\title{
Microwave oscillator based on an intrinsic BSCCO-type Josephson junction
}

\section{Pedersen, Niels Falsig; Madsen, Søren Peder}

Published in:

I E E E Transactions on Applied Superconductivity

Link to article, DOI:

10.1109/TASC.2005.850131

Publication date:

2005

Document Version

Publisher's PDF, also known as Version of record

Link back to DTU Orbit

Citation (APA):

Pedersen, N. F., \& Madsen, S. P. (2005). Microwave oscillator based on an intrinsic BSCCO-type Josephson junction. I E E E Transactions on Applied Superconductivity, (2), 948-951.

https://doi.org/10.1109/TASC.2005.850131

\section{General rights}

Copyright and moral rights for the publications made accessible in the public portal are retained by the authors and/or other copyright owners and it is a condition of accessing publications that users recognise and abide by the legal requirements associated with these rights.

- Users may download and print one copy of any publication from the public portal for the purpose of private study or research.

- You may not further distribute the material or use it for any profit-making activity or commercial gain

- You may freely distribute the URL identifying the publication in the public portal

If you believe that this document breaches copyright please contact us providing details, and we will remove access to the work immediately and investigate your claim. 


\title{
Microwave Oscillator Based on an Intrinsic BSCCO-Type Josephson Junction
}

\author{
N. F. Pedersen and S. Madsen
}

\begin{abstract}
The electrical behavior of anisotropic BSCCO single crystals is modeled by mutually coupled long Josephson junctions. For the basic fluxon modes with one fluxon per layer, the fluxons will arrange themselves in an anti phase configuration (triangular lattice) because of the mutual repulsion. We are interested in the in-phase modes (square lattice) desired for many potential applications. We consider two mechanisms (i) intrinsic locking by out of phase oscillations at the trailing edge and (ii) locking by an external high- $Q$ resonator with a resonance frequency corresponding to fluxon in-phase motion. The resulting model is a set of coupled nonlinear partial differential equations. By direct numerical simulations we have demonstrated that the qualitative behavior of the combined intrinsic Josephson junction and cavity system can be understood on the basis of general concepts of nonlinear oscillators interacting with a resonator. For some region of the parameter space it is possible to reach the desired synchronous state, making the system potentially suitable for applications. We also consider the system in the flux flow mode under a high magnetic field.
\end{abstract}

Index Terms-BSCCO, cavity, fluxons, THz oscillator.

\section{INTRODUCTION}

$\mathbf{R}$ ECENTLY attempts to fabricate a microwave oscillator based on fluxon motion in intrinsic Josephson junctions of the highly anisotropic BSCCO type have been reported [1]. The frequency range is potentially in the hundreds of gigahertz-or even terahertz range. The basic physical mechanism to be exploited is the emission of electromagnetic radiation by a Josephson fluxon when it hits an edge of the junction. In an intrinsic junction there are many junctions on top of each other and potentially the power can be enhanced by several orders of magnitude if in-phase motion of the fluxons in the different layers can be obtained. Such coherent fluxon motion is sometimes referred to as the 'square lattice' situation, and has been observed experimentally in BSCCO single crystals [1]. The difficulty in obtaining in-phase motion arises from the fact that same polarity fluxons basically repel each other and therefore would rather favor anti-phase motion. In this paper we discuss two mechanisms that may contribute to in-phase fluxon motion: (i) intrinsic nonlinearities leading to oscillations in the fluxon line shape and (ii) frequency locking using a cavity with a resonance frequency corresponding to the in-phase fluxon frequency. The latter mechanism has been observed both for small [2] and long [3]-[5] Josephson junctions. In the case to be investigated here the long Josephson junctions in the stack are both coupled to each other and individually to the

Manuscript received October 5, 2004.

The authors are with the Oersted-DTU, Section of Electric Power Engineering, The Technical University of Denmark, DK-2800 Kgs. Lyngby, Denmark.

Digital Object Identifier 10.1109/TASC.2005.850131
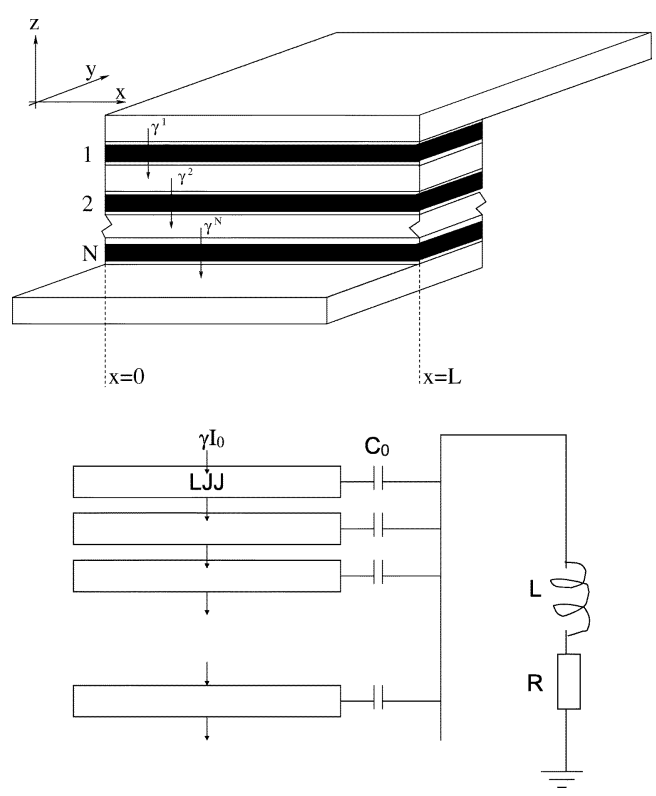

Fig. 1. Geometry of the Josephson stack (top) and schematic drawing of the BSCCO_cavity system (bottom).

cavity as shown schematically in Fig. 1. Preliminary work in that direction has recently been reported in [6]. In the last section we apply a large magnetic field through the boundary conditions and investigate the ordering of the flux lattice in the flux flow mode.

\section{THE BSCCO MODEL}

The standard model for inductively coupled stacks is a set of Partial Differential Equations coupled via the magnetic flux [7]:

$$
\mathbf{J}=\mathbf{S}^{-1} \boldsymbol{\phi}_{x x}, \quad \mathbf{S}=\left(\begin{array}{ccccc}
1 & S & & & \\
S & 1 & S & & \\
& S & 1 & S & \\
& & \ddots & \ddots & \ddots
\end{array}\right)
$$

where

$$
J^{i}=\phi_{t t}^{i}+\alpha \phi_{t}^{i}+\sin \phi^{i}-\gamma^{i}
$$

Here $\phi$ is the gauge-invariant phae difference across junction $i$, $\alpha=\left(1 / R_{j}\right) \sqrt{\hbar / 2 e I_{0} C_{j}}$ is the dissipation parameter $\left(R_{j}, I_{0}\right.$, and $C_{j}$ are the normal resistance, the critical current and the capacitance, respectively), $\gamma^{i}$ is the bias current normalized to the critical current $I_{0}$ of the individual junctions and the normalized coupling term among the junctions in the stack reads $S=\lambda_{L} / d^{\prime} \sinh \left(t / \lambda_{L}\right), d^{\prime}=d+2 \lambda_{L} \operatorname{coth}\left(t / \lambda_{L}\right)$ [7]. We only consider the case where $\gamma^{i}=\gamma^{j} \equiv \gamma$. Time is normalized to 
the inverse of the Josephson frequency $\omega_{j}=\sqrt{2 e I_{0} / \hbar C_{j}}$ and space with respect to the Josephson length $\lambda_{j}=\sqrt{\hbar / 2 e \mu_{0} d^{\prime} J^{c}}$. The resonator at one edge of the junctions (see Fig. 1) gives a boundary condition [7] expressing that the spatial derivative of the phase is equal to the current fed by the resonator, i.e.

$$
\frac{\partial \phi^{i}(L, t)}{\partial x}=\Gamma^{i}
$$

Here $\Gamma^{i}=I^{i} / I_{0}$ is the normalized current at the right hand side of the LJJ (see Fig. 1). Such current is connected to the normalized (with respect to $I_{0} / \omega_{j}$ ) charge $q$ in the capacitor of the $R L C$ circuit via the equation:

$$
\begin{aligned}
\Gamma^{i} & =-\frac{1}{N}\left(\frac{2 a}{\Omega^{2}} \frac{d^{2} q}{d t^{2}}+\frac{1}{\Omega^{2}} \frac{d^{3} q}{d t^{3}}\right)+\frac{c}{N} \phi_{t t}^{i}(L, t) \\
& =\frac{\dot{q}}{N}-\frac{c}{N^{2}} \sum_{j=1}^{N}\left(\phi_{t t}^{k}(L, t)-\phi_{t t}^{i}(L, t)\right)
\end{aligned}
$$

Here $\Omega$ is the cavity resonance frequency normalized to the Josephson frequency $\omega_{j}, \Omega^{-1}=\sqrt{N C_{0} L \omega_{j}^{2}}, Q$ its quality factor, $a=\Omega / 2 Q$ is the dissipation in the cavity and $\mathrm{c}$ is the total capacitance normalized to the Josephson capacitance, $c=N C_{0} / C_{j}$. The charge in the resonator is given by the standard linear equation for the charge of a $R L C$ circuit:

$$
\frac{d^{2} q}{d t^{2}}+2 a \frac{d q}{d t}+\Omega^{2} q=\Omega^{2} \frac{c}{N} \sum_{i=1}^{N} \phi_{t}^{i}(L, t)
$$

Eqs. (1)-(5) have been integrated with a standard fourth (sometimes fifth) order Runge-Kutta routine for the time dependence. The spatial derivative has been approximated by the two-point discrete finite difference for the first derivative and the threepoint finite difference for the second derivative.

\section{NUMERICAL RESULTS-INTRINSIC LOCKING}

Fig. 2 shows some results for an intrinsic Josephson stack without any coupling to a cavity. We have chosen the smallest nontrivial stack, $N=3$, and kept the length short in order to discover the essentials of the problem without needing too excessive calculations. In the following we will need the characteristic velocities for electromagnetic waves in the stack considered as a linear resonator. For an $N$-stack there are $N$ characteristic velocities for the $N$ different modes of propagation for linear modes. These velocities depend on the coupling parameter $\mathrm{S}$, and the simple formula for calculating these velocities may be found for example in [7]. Here we will consider only the so-called in-phase velocity $C^{+}$and the anti-phase velocity $C^{-}$, which for the case of $N=3$ are given by:

$$
C^{ \pm}=\frac{C^{0}}{\sqrt{1 \pm \sqrt{2} S}}, \quad C^{0}=\sqrt{\mu_{0} d^{\prime} C_{j}}
$$

Fig. 2 shows for $N=3$ the two most important plasma oscillation modes out of the three possible. As was demonstrated in [8] the fundamental fluxon modes lend their symmetry from the simpler plasma modes, and the figure also shows the fundamental fluxon modes together with the plasma modes of
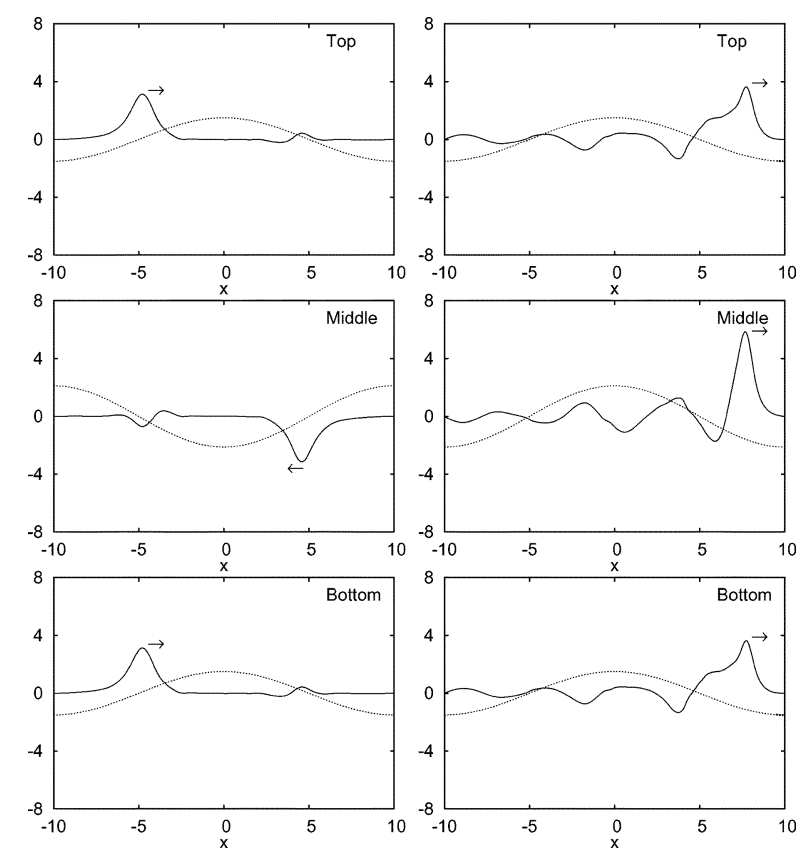

Fig. 2. Numerical solutions of anti-phase mode (left column), and in-phase mode (right column) of the 3 junction stack. The top middle and bottom junctions are shown from top to bottom in each column. The bias current is chosen to give the different modes. Full curves are fluxons and dotted curves are plasmons. Parameters used: $\alpha=0,1, S=-0,2$ and $\gamma=0,2$ (left), and 0,45 (right).

similar symmetry. Fig. 2 also defines the difference between in-phase modes ('square lattice') and anti-phase mode ('triangular lattice'). The first column of Fig. 2 shows how the anti-phase mode with essentially undisturbed fluxons manifests itself in the three junctions. The top/bottom fluxons move together but in anti-phase with the center fluxon. This is the stable and natural mode since the fluxons in the different layers repel each other. The desired mode for microwave applications is the in-phase mode seen in the second column. As can be seen, although the three fluxons move coherently, the natural fluxon pulse shape is modified by strong oscillations in the trailing edge. We note that these oscillations are in anti-phase in adjacent layers such that a maximum in one layer correspond to a minimum in the neighboring layer. Such oscillations are intrinsic to the nonlinear equations and have been noted very early in connection with soliton locking [9]. Here it was noted that with 2 solitons (fluxons) on the same line, the solitons could bunch by a mechanism in which a soliton was trapped in the potential created by trailing oscillations of the other soliton. Also similar oscillations have been seen in connection with the so-called Cerenkov oscillations in Josephson stacks [10]. The appearance of such anti-phase oscillations is a dynamic effect and is the mechanism that keep fluxons phase locked in spite of their natural repulsion. This mechanism exists even for the stack without a cavity; but as we shall see later, a resonant cavity represents another locking mechanism that may enhance in-phase motion. The third mode (not shown) has the top and bottom fluxons and plasmons in anti-phase and the middle junction has neither a fluxon or a plasma oscillation [8].

To get an understanding of the nature of the trailing edge oscillations we note that for $N=3$ an approximate analytical 


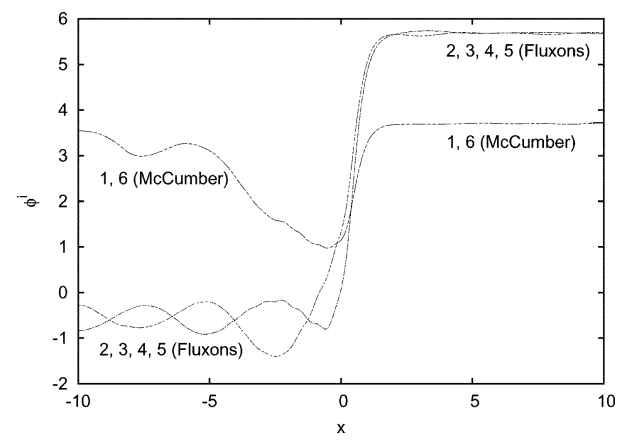

Fig. 3. $N=6$. Top and bottom junctions have switched to the McCumber curve.

expression can be derived [8]. We assume the phases of the top/bottom and middle junctions respectively are expressed by

$$
\begin{aligned}
& \phi^{1}=\phi+\delta \phi=\phi^{3} \\
& \phi^{2}=\phi-\kappa \delta \phi
\end{aligned}
$$

Inserting this in (1) we find

$$
\delta \phi \approx e^{\kappa \xi}\left(A_{1} \cos (\omega \xi)+A_{2} \sin (\omega \xi)\right), \quad \kappa=\sqrt{2}
$$

with $\xi \equiv x-x_{0}-v t$ and where $A_{1}$ and $A_{2}$ are some unknown constants. A plot showing the above formula together with a numerical solution of the full system can be seen in [8]. Such damped anti-phase oscillations can also be seen in the second column of Fig. 2. The analytical result shows what happens if we have the bunched state and then introduces some small difference between the top/bottom and center junction. The system reacts with anti-phase oscillations in the two junctions trying to preserve fluxon locking, for example with $\phi^{2}$ being trapped in a local minimum of $\phi^{1}$. The appearance of an amplitude of $\sqrt{2}$ in the amplitude of $\phi^{2}$ is a natural consequence of the middle layer interacting with both the top and bottom layers. Several authors have investigated this locking scenario i.e. for example [11].

The role of the oscillations discussed for $N=3$ are generic for the locking mechanism leading to in-phase fluxon motion.

As discussed above the basic idea is that the fluxons get locked in the in-phase configuration through the trailing edge oscillations. Since the fluxons in the neighboring layers naturally repel each other there is a competition between the two mechanisms. With more and more layers in the stack it was found in [8] that particularly the top and bottom junctions has a tendency to switch to the McCumber curve and thus loose the fluxons in the outer layers. Such an example is shown in Fig. 3 for a 6 layer stack. Fluxons in junctions 2,3,4,5 are in phase-locked together by the anti phase trailing oscillations (2, 5 and 3, 4 are identical for reasons of symmetry). In layers 1, 6 the anti-phase trailing oscillations were not sufficient to lock these fluxons, and the top and bottom junctions switched to finite voltage. (For clarity we show here the phase $\phi$ rather than the voltage $\phi_{t}$ ). It is however important to note that the fluxon structure from the internal junctions are repeated in the switched top/bottom junctions. Thus in the voltage $\phi_{t}$ there would still be an in-phase voltage pulse-although with a slightly reduced amplitude. When the total voltage is found by adding all the voltage pulses from all the junctions, we find that

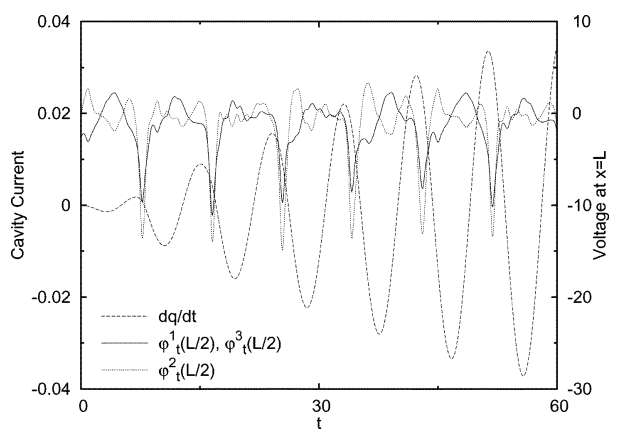

Fig. 4. $\quad \mathrm{N}=3$. Dynamics of the fluxon system charging the cavity. The in-phase pulses from the $\mathrm{N}=3$ stack inject charge into the cavity close to its resonance frequency. A sinusoidal cavity current builds up (dashed curve) eventually phase locking the junctions to its (resonance) frequency.

the switching of the top and bottom junctions has negligible effect on the amplitude of the total voltage pulse, and thus on the potential power to be generated in the oscillator mode [12].

\section{NUMERICAL RESULTS-LOCKING BY EXTERNAL RESONATOR}

Besides the anti-phase trailing oscillations discussed above the other mechanism for in-phase locking of the fluxons in the stack is the interaction with a cavity when the fluxon frequency is close to the resonator frequency. The basic idea is that the fundamental oscillators (the fluxons moving back and forth in the individual junction layers) emit pulsed radiation at a frequency close to the resonance frequency of the cavity. At each collision some small amount of power-depending on the coupling coefficient, determined essentially by $C_{0}$-is transferred to the cavity. The current waveform in the (linear) cavity is essentially sinusoidal, and the current amplitude builds up over many fluxon oscillation periods until a power balance is obtained, see Fig. 4. In this situation the power transferred from the fluxons to the cavity in each oscillation period is equivalent to the power transferred from the cavity to the junctions in the same period. When the frequency of the oscillators is close enough to the resonance frequency of the cavity, the amplitude of the cavity oscillations become large enough to furnish the clock that forces the junction fluxon oscillators to phase-lock to the cavity resonance frequency. The mechanism is rather equivalent to the case of applying an external microwave signal to phase lock all the junctions in a stack [13], [14].

If the junction parameters are such that intrinsically the in-phase motion is favored at a frequency close to the cavity resonance frequency, the cavity-junction interaction will stabilize the fluxon in-phase motion and contribute even further to the build up of power in the cavity. We note as a general feature of our system that it is an unusual variant of the classical problem of a nonlinear oscillator coupled to a linear resonator. Here all the individual oscillators are each coupled to the cavity. In addition the individual oscillators are coupled to each other by inductive coupling, defining a number of different intrinsic oscillation modes. The competition between the two different types of coupling of the junctions will be essential for the outcome of the dynamics, and the possibilities for utilizing the combined system for applications. 


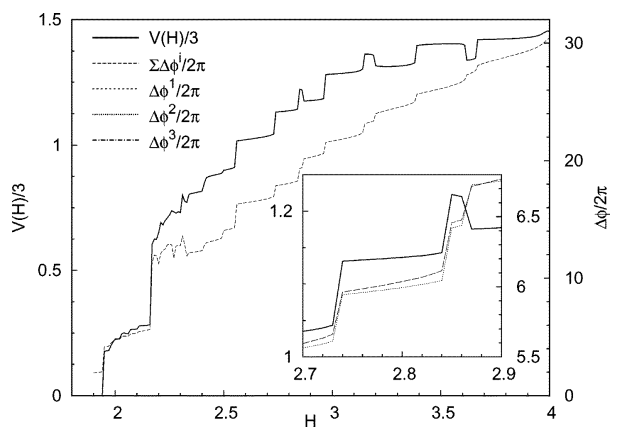

Fig. 5. The magnetic field dependence of the 3 junction stack. The inset shows the phase-behavior of the individual junctions and the average voltage near $H=$ 2.8. Parameters used: $N=3, L=20, S=-0.1, \alpha=0.1$ and $\gamma=-0.15$.

\section{Numerical Results-Magnetic Field DEPENDENCE AND FLUX FLOW}

Some of the recent experimental results on BSCCO have been obtained by applying a magnetic field while having a constant bias current [15]. Fig. 5 shows the effect of applying a constant (with respect to time) magnetic field to a 3 stack with a bias current of $\gamma=-0.15$. The average flux flow voltage per junction is shown. For low values of the magnetic field there is a transition to a state in which fluxons enter the center junction. When $H$ is about 2.2 there is another transition to a state in which fluxons enter all three junctions in the stack. In this so-called flux flow state fluxons enter from one side and leave from the other-driven by the applied magnetic field. Also shown (right scale) is the total phase shift from one side of the stack to the other, $\phi^{i}(L)-\phi^{i}(0)$, which is related to the number of fluxons present in the stack. The jumps correspond to the entering of fluxons. To help understanding the entering of fluxons, the inset shows, as an example, the details near $H=2.8$.

We note, that although the flux flow voltage looks somewhat erratic, it is reproducible under the changing of initial conditions, step size etc. but there is a small hysteresis in the system. From the time pictures (not shown) we can see that the fluxon lattice is triangular with the top and bottom fluxons in phase. The symmetry of one plus two can clearly be seen in the total number of fluxons (right scale in Fig. 5), however the inset shows that both top/bottom and center phase shift is rather similar, indicating that the triangular lattice is rather stiff. On the other hand, the rate of change of both flux entry and flux flow voltage change significantly with the magnetic field and also with bias current. Accordingly we also made a calculation of the derivative with respect to the bias current, i.e. the flux flow resistance. It shows clear and regular resonances, instead of steps, corresponding to fluxon entry. The appearance of the resonances in the flux flow resistance as a function of the magnetic field is in general agreement with measurements on BSCCO single crystals [1], [15], although we only observed triangular lattice, not square lattice. We suspect the reason for not finding the square lattice is that we have not yet identified the proper parameters.

\section{CONCLUSION}

The system consisting of a stack of Josephson junctions has been investigated with the purpose of understanding the in-phase modes. Both the intrinsic origins and the coupling of the stack to a cavity were considered. We find that anti-phase oscillations in the trailing edges of neighboring fluxons play an essential role in the locking process. With a resonator we find that if it has a resonance frequency corresponding to the in-phase intrinsic resonance of the stack, large amounts of power can be coupled to the cavity. We investigated the fluxon ordering in a magnetic field and found only the anti-phase ordering (triangular lattice). This part is not conclusive since we investigated only a limited number of parameter values. The BSCCO system with in-phase fluxon ordering has a potential for practical applications in microwave generation using high $T_{c}$ BSCCO single crystals at hundreds of Gigahertz.

\section{ACKNOWLEDGMENT}

The support from the Danish STVF program "New superconductors" is acknowledged. Conversations and collaboration with Giovanni Filatrella have contributed significantly to this work.

\section{REFERENCES}

[1] T. Hatano, H. B. Wang, S. Kim, S. Urayama, S. Kawakami, S.-J. Kim, M. Nagao, K. Inomata, Y. Takano, T. Yamashita, and M. Tachiki, IEEE Trans. on Appl. Supercond., vol. 2, no. 5, Jun. 2004.

[2] P. Barbara, A. B. Cawthorne, S. V. Shitov, and C. J. Lobb, "Stimulated emission and amplification in Josephson junction array," Phys. Rev. Lett., vol. 82, p. 1963, 1999

[3] S. Pagano, R. Monaco, and G. Costabile, "Microwave oscillator using array of long Josephson junctions," IEEE Trans. Magn., vol. 25, p. 1080, 1989.

[4] N. Groenbech-Jensen, R. D. Parmentier, and N. F. Pedersen, "Phase-locking of flucon oscillations in long Josephson junctions coupled through a resonator," Phys. Lett. A, vol. 142, p. 427, 1989.

[5] G. Filatrella, G. Rotoli, N. Groenbech-Jensen, R. D. Parmentier, and N F. Pedersen, "Model studies of long Josephson junction arrays coupled to a high-Q resonator," J. Appl. Phys., vol. 72, p. 3179, 1992.

[6] S. Madsen, G. Filatrella, and N. F. Pedersen, "Interaction between a BSCCO-type intrinsic Josephson junction and a microwave cavity," The European Physical Journal B, vol. 40, no. 2, pp. 209-215, 2004.

[7] S. Sakai, P. Bodin, and N. F. Pedersen, "Fluxons in thin film superconductor-insulator superlattices," J. Appl. Phys., vol. 73, p. 2411, 1993.

[8] S. Madsen and N. F. Pedersen, "Fluxon modes in stacked Josephson junctions: the role of linear modes," Phys. Rev. B, vol. 69, p. 064507 , 2004.

[9] K. Nakajima, Y. Sawada, and Y. Onodera, "Nonequlibrium stationary coupling of solitons," J. Appl. Physics, vol. 46, p. 5272, 1975.

[10] J. Zitsmann, A. V. Ustinov, M. Levitchev, and S. Sakai, "Super-relativistic fluxon in a Josephson multiplayer: experiment and simulation," Phys. Rev. B, vol. 66, p. 064 527, 2002.

[11] C. Gorria, P. L. Christiansen, Y. B. Gaididei, V. Muto, N. F. Pedersen, and M. P. Soerensen, "Fluxons and their interactions in three stacked Josephson junctions," Phys. Rev. B, vol. 68, p. 035 415, 2003.

[12] N. F. Pedersen and S. Madsen, "Fluxon dynamics in BSCCO-microwave emission," Supercond. Sci. Technol., vol. 17, p. S117, 2004.

[13] H. B. Wang, P. H. Wu, and T. Yamashita, "Terahertz responses of intrinsic Josephson junctions in high $\mathrm{T}_{C}$ superconductors," Phys. Rev Lett., vol. 87, p. $107002,2001$.

[14] S. Madsen and N. Grønbech-Jensen, "Microwave induced fluxon bunching in weakly coupled Josephson junctions," Phys. Rev. B, 2004.

[15] H. B. Wang and T. Hatano, "NIMS," unpublished, 2004. 